\title{
FEEDBACK INSTABILITY IN A BOUNDARY-LAYER FLOW OVER ROUGHNESS
}

\author{
S. N. TIMOSHIN
}

Abstract. Linear stability of an incompressible triple-deck flow over a wall roughness is considered for disturbances of high frequency. The wall roughness consists of two relatively short obstacles placed far apart on an otherwise flat surface. It is shown that the flow is unstable to feedback or global mode disturbances. The feedback loop is formed by algebraically decaying disturbances propagating upstream and weakly growing Tollmien-Schlichting waves travelling downstream and as such represents an interaction between modes from continuous and discrete spectra of the corresponding parallelflow problem. An example of growth rate calculation for a specific roughness is considered.

§1. Introduction. Tollmien-Schlichting instabilities near the lower branch of a neutral curve for a laminar boundary layer in an incompressible fluid are governed by the equations of viscous-inviscid interaction (tripledeck equations). These equations are formally valid at asymptotically large Reynolds numbers in the flow, and they describe near-wall motion of the fluid in a local region at a chosen downstream location on a solid boundary of small curvature. Depending on the context, a non-trivial flow within the triple-deck can be due to an isolated wall roughness, or a self-induced separation, or, in unsteady flows, to a travelling-wave or a pulse disturbance introduced by various means, as reviewed, for example, in $[3,7,8]$. Within the triple-deck formulation, the base, unperturbed, steady flow along a flat surface is unstable to infinitesimal travelling-wave disturbances [5, 11]. The spectrum of instability consists of a single mode and includes a lower-branch neutral point, a range of most highly amplified waves, and a semi-infinite range of short unstable waves with finite growth rates. Despite the fact that the upper-branch cut-off of the instability is not captured by the triple-deck model, the initial value problem for the triple-deck equations proves to be well posed. It was also found that the base triple-deck flow exhibits convective (rather than absolute) instability, i.e., the amplified part of a compact disturbance introduced in the flow at a finite time will be swept away from the flow region leaving no growing disturbances behind [4].

The aim in the present paper is to show that a weak, distributed spatial inhomogeneity in the triple-deck flow, for definiteness due to a pair of isolated obstacles placed sufficiently far apart, may be sufficient to trigger a form of absolute instability or unstable global mode in conventional terminology (see, e.g., [2]). The specific form of absolute instability considered here relies on a feedback loop sustained by Tollmien-Schlichting waves growing in 
amplitude as they propagate downstream, and inviscid pressure waves decaying algebraically as they spread through the inviscid part of the flow. In terms of the governing equations it is a link between the parabolic/hyperbolic properties of the wave system in the boundary layer and the ellipticity introduced through the viscous-inviscid interaction that alters the spectral characteristics of the flow. The link relies on the presence of regions of spatial inhomogeneity, here in the form of wall-mounted obstacles. It is important to note a fundamental difference between the non-local interaction mechanism in this work and the effect of local, short-wave, destabilisation observed near concentrated wall roughness with strongly deformed velocity profiles $([\mathbf{6}, \mathbf{1 0}])$.

The analysis in this work is restricted to disturbances in the short-wave part of the Tollmien-Schlichting spectrum. As a result we shall derive certain sufficient conditions for the origin of feedback modes. The task of establishing conditions for the first appearance of non-local instabilities is beyond the scope of this study.

§2. Problem formulation and linear spectrum. In the standard for the boundary-layer theory notation, the triple-deck formulation for the flow over a wall roughness can be written as a coupled system of near-wall viscous flow and outer inviscid-flow equations:

$$
\begin{array}{cl}
\frac{\partial u}{\partial t}+u \frac{\partial u}{\partial x}+v \frac{\partial u}{\partial y}=-\frac{\partial p}{\partial x}+\frac{\partial^{2} u}{\partial y^{2}}, & \frac{\partial u}{\partial x}+\frac{\partial v}{\partial y}=0, \\
u=y+A(x, t)+f(x) \text { as } y \rightarrow \infty, & u=v=0 \text { at } y=0, \\
\frac{\partial^{2} \phi}{\partial x^{2}}+\frac{\partial^{2} \phi}{\partial z^{2}}=0,\left.\quad \frac{\partial \phi}{\partial z}\right|_{z=0}=-\frac{\partial A}{\partial x}, & \left.\frac{\partial \phi}{\partial x}\right|_{z=0}=-p(x, t) .
\end{array}
$$

Here all variables are non-dimensional and scaled in the usual fashion, $u(x, y, t), v(x, y, t), p(x, t)$ denote the streamwise (along the $x$-axis) and normal (along the $y$-axis) velocity components and pressure in the near-wall viscous layer respectively, $A(x, t)$ is the negative displacement thickness, and $t$ is time. The function $f(x)$ specifies the wall roughness, with Prandtl's transposition used to simplify the wall conditions. In the inviscid-flow formulation, $\phi(x, z, t)$ is the velocity potential. The flow is entirely two-dimentional.

Steady-state (or mean flow) solutions of (2.1)-(2.3) exist provided that the roughness is not excessively large, with $u=u_{m}(x, y), v=v_{m}(x, y), p=p_{m}(x)$ and $A=A_{m}(x)$ determined from the following boundary-value problem:

$$
\begin{gathered}
u_{m} \frac{\partial u_{m}}{\partial x}+v_{m} \frac{\partial u_{m}}{\partial y}=-p_{m}^{\prime}+\frac{\partial^{2} u_{m}}{\partial y^{2}}, \quad \frac{\partial u_{m}}{\partial x}+\frac{\partial v_{m}}{\partial y}=0, \\
u_{m}=y+A_{m}(x)+f(x) \text { as } y \rightarrow \infty, \quad u_{m}=v_{m}=0 \text { at } y=0, \\
u_{m} \rightarrow y \text { as }|x| \rightarrow \infty, \quad p_{m}(x)=\frac{1}{\pi} \int_{-\infty}^{\infty} A_{m}^{\prime}(s) \frac{d s}{x-s},
\end{gathered}
$$


where the prime designates the derivative. The principal value integral in (2.6) is a familiar result in the viscous-inviscid interaction theory obtained from solving (2.3) for the time-independent potential, $\phi=\phi_{m}(x, z)$ say, with the mean-flow decay condition imposed upstream and downstream.

The linear stability problem for the mean flow above can be posed as an eigen-value problem, considering small disturbances of amplitude $\delta \ll 1$ of (complex-valued in general) frequency $\omega$ superimposed on the solution of (2.4)-(2.6). Hence

$$
\begin{aligned}
\{u, v, p, A, \phi\}= & \left\{u_{m}, v_{m}, p_{m}, A_{m}, \phi_{m}\right\} \\
& +\delta \exp (-i \omega t)\left\{u_{u}(x, y), v_{u}(x, y), p_{u}(x), A_{u}(x), \phi_{u}(x, z)\right\}+O\left(\delta^{2}\right),
\end{aligned}
$$

including complex conjugate terms where necessary. Then, neglecting terms of order $\delta^{2}$,

$$
\begin{gathered}
-i \omega u_{u}+\frac{\partial\left(u_{m} u_{u}\right)}{\partial x}+v_{m} \frac{\partial u_{u}}{\partial y}+v_{u} \frac{\partial u_{m}}{\partial y}=-p_{u}^{\prime}+\frac{\partial^{2} u_{u}}{\partial y^{2}}, \quad \frac{\partial u_{u}}{\partial x}+\frac{\partial v_{u}}{\partial y}=0, \\
u_{u} \rightarrow A_{u}(x) \text { as } y \rightarrow \infty, \quad u_{u}=v_{u}=0 \text { at } y=0, \\
\frac{\partial^{2} \phi_{u}}{\partial x^{2}}+\frac{\partial^{2} \phi_{u}}{\partial z^{2}}=0,\left.\quad \frac{\partial \phi_{u}}{\partial z}\right|_{z=0}=-A_{u}^{\prime}(x),\left.\quad \frac{\partial \phi_{u}}{\partial x}\right|_{z=0}=-p_{u}(x) .
\end{gathered}
$$

The appropriate boundary condition as $|x| \rightarrow \infty$ is a radiation condition on the instability waves present in the system (2.8)-(2.10). Sufficiently far from the roughness, the steady-state in (2.4)-(2.6) reduces to $u_{m}=y, v_{m}=p_{m}=A_{m}=0$, and hence a travelling-wave form can be taken for the disturbance in (2.8)(2.10), with the $x$-dependence in all functions specified explicitly as $\exp (i k x)$. A non-trivial solution for the disturbance can then be found provided the wavenumber $k$ and frequency $\omega$ satisfy the dispersion relation

$$
A i^{\prime}\left[-i \omega(i k)^{-2 / 3}\right]=(i k)^{1 / 3} k \int_{0}^{\infty} A i\left[s-i \omega(i k)^{-2 / 3}\right] d s,
$$

where $A i$ is the Airy function, $|\arg (i k)|<\pi$, and the real part of $k$ is assumed positive without loss of generality. As is now well known, the roots of (2.11) written as $k=k_{n}(\omega), n=1,2, \ldots$, with real frequency $\omega$ or, conversely, $\omega=\omega_{n}(k)$ with real wavenumber $k$, form a countable discrete spectrum of the linear triple-deck problem. Only one of the modes from this spectrum contains instability waves. For short unstable waves, of typical wavelength of order $\varepsilon \ll 1$ say, the dispersion relation (2.11) yields

$$
\begin{gathered}
\omega=\varepsilon^{-2} \omega_{0}+\omega_{1}+o(1), \quad k=\varepsilon^{-1} k_{0}+\varepsilon k_{1}+o(\varepsilon), \\
\omega_{0}=k_{0}^{2}, \quad \omega_{1}=e^{i \pi / 4}+2 k_{0} k_{1} .
\end{gathered}
$$

For both temporal (real $k$ ) and spatial (real $\omega$ ) forms of instability, the short waves prove to be neutral at leading order. The radiation condition mentioned 
above can therefore be formulated as the condition of absence of these nearneutral waves in the solution of (2.8)-(2.10) as $x \rightarrow-\infty$. Physically, this corresponds to eliminating the incoming instability wave in the flow upstream of the roughness. Hence the frequency $\omega$ in (2.8)-(2.10) is an eigenvalue to be determined as part of the solution. Solutions of the disturbance equations (2.8)-(2.10) with the imaginary part $\omega_{i}>0$ will correspond to global or absolute instabilities growing in the entire flow field sorrounding the roughness, as opposed to convective instability of the waves contained in the spectrum of the flat-plate flow (2.11).

§3. Feedback instability. In addition to the discrete wave spectrum described by (2.11), the formulation (2.8)-(2.10) also contains disturbances of a continuum spectrum. They are interpreted conventionally as pressure waves appearing in the solution of the Laplace equation (2.10). We aim to show that an interaction between discrete and continuous spectra taking place in the regions of spatial inhomogeneity gives rise to a feedback loop which in turn leads to self-sustained growth of disturbances. To this end the roughness will be represented by a pair of isolated obstacles, each with typical length comparable to the discrete wavelength $O(\varepsilon)$ to begin with, and placed at a large distance $L$ from each other. In what follows it is convenient (although not essential) to assume the obstacle height to be of $O\left(\varepsilon^{1 / 3}\right)$. Specifically, if

$$
f(x)=\varepsilon^{1 / 3}\left[F_{1}\left(\frac{x}{\varepsilon}\right)+F_{2}\left(\frac{x-L}{\varepsilon}\right)\right], \quad F_{1}( \pm \infty)=F_{2}( \pm \infty)=0,
$$

then, to leading order, the solution of (2.4)-(2.6) can be written as

$$
\left\{u_{m}, v_{m}, p_{m}, A_{m}\right\}=\left\{\varepsilon^{1 / 3} u_{m 1}, \varepsilon^{-1 / 3} v_{m 1}, \varepsilon^{2 / 3} p_{m 1}, \varepsilon^{5 / 3} A_{m 1}\right\}+\cdots,
$$

with the governing equations for the base flow around each of the obstacles of the form

$$
\begin{gathered}
u_{m 1} \frac{\partial u_{m 1}}{\partial X_{1,2}}+v_{m 1} \frac{\partial u_{m 1}}{\partial y_{1}}=-p_{m 1}^{\prime}+\frac{\partial^{2} u_{m 1}}{\partial y_{1}^{2}}, \quad \frac{\partial u_{m 1}}{\partial X_{1,2}}+\frac{\partial v_{m 1}}{\partial y_{1}}=0, \\
u_{m 1}=y_{1}+F_{1,2}\left(X_{1,2}\right) \text { as } y_{1} \rightarrow \infty, \quad u_{m 1}=v_{m 1}=0 \text { at } y_{1}=0, \\
u_{m 1} \rightarrow y_{1} \text { as }\left|X_{1,2}\right| \rightarrow \infty .
\end{gathered}
$$

Here the $O(1)$ independent variables are: for the upstream obstacle, $y_{1}=$ $y \varepsilon^{-1 / 3}$ and $X_{1}=x \varepsilon^{-1}$, for the downstream obstacle, $y_{1}$ and $X_{2}=(x-L) \varepsilon^{-1}$, with the scaled obstacle shapes $F_{1}\left(X_{1}\right)$ and $F_{2}\left(X_{2}\right)$, respectively. As a result, the mean flow around each of the obstacles is in the so-called condensed-layer regime, and these two parts of the steady flow can be regarded as independent of each other.

Solutions of the disturbance equations (2.8)-(2.10) can be constructed considering three separate flow regions in the $x$-direction. Using $\varepsilon$ as a key small parameter, the frequency is sought in the form $\omega=\varepsilon^{-2} \omega_{0}+\omega_{1}+o(1)$, similar to (2.12). Then, in the first region covering the vicinity of the downstream obstacle, we assume (subject to an ultimate matching of the 
solutions in all regions) that the leading order disturbance is a wave from the discrete spectrum of the parallel-flow problem with a typical amplitude $C_{0}$, and hence in the region $\left(X_{2}, y_{1}\right)=O(1)$ we have

$$
\begin{aligned}
u_{u} & =C_{0}\left[e^{i k_{0} X_{2}}+\varepsilon^{4 / 3} U_{1}\left(X_{2}, y_{1}\right)+\cdots\right], \\
v_{u} & =C_{0}\left[-\varepsilon^{-2 / 3} y_{1} i k_{0} e^{i k_{0} X_{2}}-e^{i k_{0} X_{2}}(-i)^{1 / 2}+\varepsilon^{2 / 3} V_{1}\left(X_{2}, y_{1}\right)+\cdots\right], \\
p_{u} & =C_{0}\left[\varepsilon^{-1} k_{0} e^{i k_{0} X_{2}}+\varepsilon^{1 / 3} P_{1}\left(X_{2}\right)+\cdots\right], \\
A_{u} & =C_{0}\left[e^{i k_{0} X_{2}}+\varepsilon^{4 / 3} A_{1}\left(X_{2}\right)+\cdots\right], \\
\phi_{u} & =C_{0}\left[e^{k_{0}\left(i X_{2}-Z\right)}+\varepsilon^{4 / 3} \Phi_{1}\left(X_{2}, Z\right)+\cdots\right],
\end{aligned}
$$

with $Z=z / \varepsilon=O(1)$ in (3.10). Note that the main interaction between the incoming discrete wave and the obstacle takes place in the layer of thickness $y_{1}=O(1)$ which is thicker than the Stokes viscous layer for the wave. To the order considered here, the effect of the Stokes layer is contained in the second term in (3.7). On substituting (3.6)-(3.8) into (2.8)-(2.10) we find immediately that $\omega_{0}=k_{0}^{2}$ from the leading-order solution, and then, at the next order in $\varepsilon$, that

$$
-i \omega_{0} U_{1}+\frac{\partial}{\partial X_{2}}\left(u_{m 1} e^{i k_{0} X_{2}}\right)-i k_{0} y_{1} e^{i k_{0} X_{2}} \frac{\partial u_{m 1}}{\partial y_{1}}=-\frac{\partial P_{1}}{\partial X_{2}} .
$$

Using the boundary condition, $U_{1}\left(y_{1} \rightarrow \infty\right) \rightarrow A_{1}$, and solving for the potential $\Phi_{1}$ in the usual manner, we then find that the pressure induced as a result of the wave-obstacle interaction can be written as a Fourier integral,

$$
P_{1}\left(X_{2}\right)=\frac{1}{2 \pi} \int_{-\infty}^{\infty} e^{i k X_{2}} \frac{k|k|}{k_{0}^{2}-k|k|}\left(\int_{-\infty}^{\infty} F_{2}(S) e^{i\left(k_{0}-k\right) s} d s\right) d k,
$$

with the contour for the first integration taken below the pole at $k=k_{0}$.

It is clear that, in the process of interaction with the obstacle, the incoming wave is scattered into an additional wave of the discrete spectrum propagating downstream (due to the residue contribution of the pole) and an algebraically decaying upstream and downstream pressure wave (due to a non-analyticity of the integrand in the complex plane $k$ ). The pressure wave is formed by disturbances of the continuous spectrum, with the result that

$$
P_{1}\left(X_{2}\right)=P_{10}\left|X_{2}\right|^{-3}+\cdots, \quad \text { as } X_{2} \rightarrow-\infty,
$$

as is typical for a localized obstacle. The value of the coefficient $P_{10}$ depends on the obstacle shape. The induced pressure forces a viscous sublayer flow with $\bar{y}=y / \varepsilon=O(1)$ and the streamwise velocity of the form

$$
\begin{aligned}
u_{u}= & C_{0}\left[e^{i k_{0} X_{2}}\left(1-\exp \left(-\left(-i \omega_{0}\right)^{1 / 2} \bar{y}\right)\right)\right. \\
& \left.+\varepsilon^{4 / 3} \frac{3}{i \omega_{0}} P_{10} X_{2}^{-4}\left(1-\exp \left(-\left(-i \omega_{0}\right)^{1 / 2} \bar{y}\right)\right)+\cdots\right] .
\end{aligned}
$$

In the potential field upstream, this corresponds to an algebraic decay, $\Phi_{1}=-P_{10} r^{-2} \cos (2 \vartheta) / 2+\cdots$ in polar coordinates with $X_{2}=r \cos \vartheta \rightarrow-\infty$. 
The second characteristic region in the flow is near the upstream obstacle. Here the disturbance is dominated by the pressure wave generated downstream, hence

$$
\begin{gathered}
u_{u}=C_{1}\left[1+\varepsilon^{4 / 3} U_{1}\left(X_{1}, y_{1}\right)+\cdots\right], \quad v_{u}=C_{1}\left[\varepsilon^{2 / 3} V_{1}\left(X_{1}, y_{1}\right)+\cdots\right], \\
\frac{\partial p_{u}}{\partial x}=C_{1}\left[i k_{0}^{2} \varepsilon^{-2}+\varepsilon^{-2 / 3} P_{1}^{\prime}\left(X_{1}\right)+\cdots\right], \\
A_{u}=C_{1}\left[1+\varepsilon^{4 / 3} A_{1}\left(X_{1}\right)+\cdots\right],
\end{gathered}
$$

where $C_{1}$ is a constant to be determined shortly. The governing equation for the velocity $U_{1}$,

$$
-i \omega_{0} U_{1}+\frac{\partial u_{m 1}}{\partial X_{1}}=-P_{1}^{\prime},
$$

and the boundary condition, $U_{1}\left(y_{1} \rightarrow \infty\right) \rightarrow A_{1}$, give the following result:

$$
U_{1}\left(y_{1} \rightarrow \infty\right)=-\frac{1}{2 \pi} \int_{-\infty}^{\infty} e^{i k X_{1}} \frac{k}{k|k|-k_{0}^{2}}\left(\int_{-\infty}^{\infty} e^{-i k s} F_{1}(s) d s\right) d k .
$$

The contour of integration in the $k$-plane must be chosen in accordance with the radiation condition for the instability wave which in the present context requires the contour to pass under the pole at $k=k_{0}$. Hence downstream, as $X_{1} \rightarrow \infty$, the instability wave emerges with the amplitude in the streamwise velocity of the form

$$
u_{u}=\cdots+C_{1} \varepsilon^{4 / 3}\left(-\frac{i}{4}\right) \bar{F}_{1}\left(k_{0}\right) e^{i k_{0} X_{1}}+\cdots,
$$

where

$$
\bar{F}_{1}\left(k_{0}\right)=\int_{-\infty}^{\infty} e^{-i k_{0} s} F_{1}(s) d s
$$

is the Fourier transform of the upstream obstacle shape evaluated at the pole.

The third region extends between the obstacles and has a total length $L$. Here the effect of the roughness on the disturbance can be neglected, and hence the discrete wave (3.20) develops in accordance with the wavenumberfrequency relations (2.12)-(2.13). This wave reaches the second obstacle with the amplitude

$$
C_{0}=C_{1} \varepsilon^{4 / 3}\left(-\frac{i}{4}\right) \exp \left(i\left(k_{0} \varepsilon^{-1}+\frac{\omega_{1}-e^{i \pi / 4}}{2 k_{0}} \varepsilon\right) L\right) \bar{F}_{1}\left(k_{0}\right),
$$

as follows from (3.6) and (3.20), with a frequency shift proportional to $\omega_{1}$ taken into account.

It remains to match the pressure wave generated at the downstream obstacle in (3.13) with that acting on the upstream obstacle in (3.16). The result can be written as

$$
3 \varepsilon^{16 / 3} C_{0} P_{10} L^{-4}=C_{1} i k_{0}^{2} .
$$


Eliminating $C_{0}$ and $C_{1}$ between (3.22), (3.23), we arrive at the dispersion relation for the feedback modes,

$$
\frac{3}{4} \varepsilon^{20 / 3} L^{-4} k_{0}^{-2} \exp \left(i\left(k_{0} \varepsilon^{-1}+\frac{\omega_{1}-e^{i \pi / 4}}{2 k_{0}} \varepsilon\right) L\right) P_{10} \bar{F}_{1}\left(k_{0}\right)=-1 .
$$

§4. An example of mode analysis. For a particular illustration we consider a pair of Gaussian humps, $F_{1}\left(X_{1}\right)=F_{10} \exp \left(-X_{1}^{2} / \beta^{2}\right), \quad F_{2}\left(X_{2}\right)=$ $F_{20} \exp \left(-X_{2}^{2} / \alpha^{2}\right)$, with positive constant height and width parameters, $F_{10}, F_{20}, \alpha, \beta$. Substitution into (3.12), (3.21) then gives $P_{10}=2 i k_{0}^{-2} \alpha \sqrt{\pi}$ $\exp \left(-\alpha^{2} k_{0}^{2} / 4\right) F_{20}, \bar{F}_{1}\left(k_{0}\right)=F_{10} \beta \sqrt{\pi} \exp \left(-\beta^{2} k_{0}^{2} / 4\right)$. The dispersion relation (3.24) takes the form

$$
\widehat{F} L^{-4} \varepsilon^{20 / 3} \exp \left[i L\left(k_{0} \varepsilon^{-1}+\varepsilon\left(\omega_{1}-e^{i \pi / 4}\right) /\left(2 k_{0}\right)\right)\right]=i,
$$

where

$$
\widehat{F}=\frac{3}{2} F_{10} F_{20} k_{0}^{-4} \sqrt{\alpha \beta} \exp \left(-\left(\alpha^{2}+\beta^{2}\right) k_{0}^{2} / 4\right) .
$$

We recall that, by construction, $\left|\omega_{1}\right| \ll \omega_{0} / \varepsilon^{2}=k_{0}^{2} / \varepsilon^{2}$. Then, with $\omega_{1 r}=\omega_{1 r}+$ $i \omega_{1 i}$, the real part of (4.1) yields

$$
\frac{k_{0} L}{\varepsilon}=\frac{\pi}{2}(1+4 n)[1+o(1)],
$$

with (4.6) needed again in the intermediate case.

Finally, if the shape of the obstacles is fixed but the distance between them varies, then the disturbance with a fixed mode number $n$ is stable for smaller $L$ but then passes through a neutral point and becomes unstable as $L$ increases, with the growth rate approching a finite limit, $\omega_{i} \rightarrow 1 / \sqrt{2}$ as $L \rightarrow \infty$. This means that an upper branch cut-off of the instability is not captured by the present approximation and probably requires the overall frequency to become $O(1)$, as follows from (4.5) with $n$ fixed and $L$ increasing.

Similar conclusions can be drawn for other smooth obstacle shapes, with only minor modifications required in the case of less smooth obstacles.

§5. Discussion. The procedure adopted in this paper in constructing the feedback modes involves simultaneous solution of the receptivity problem on the first (upstream) obstacle and the wave scattering problem on the second (downstream) obstacle. In the short-wave approximation the two problems can be treated separately, so that matching of the two solutions reduces to a straightforward comparison of the amplitude coefficients for the instability and pressure waves in the two local regions. It is essential of course that, in the intermediate region between the obstacles, the instability wave changes in amplitude sufficiently fast to counter the algebraic decay of the pressure waves in the potential flow region.

In a more general context, we have shown that for the particular flow in this paper the conditions of global instability are not related to the existence of a range of local absolute instability as required in the theorem proposed in [1] for slowly varying flows. Note that in this problem global instability signifies 
growth in time for disturbances which are not localized in space, on account of the infinite flow domain. It is also worth noting that instability persists even when the underlying wave component is short relative to the obstacle lengthscale (see solutions with $k_{0} \gg \sigma^{1 / 2}$ in $\S 4$ ). In such cases one would expect the stability analysis within a quasi-parallel (frozen profile) approximation to be applicable, with an immediate conclusion of convective instability for the entire flow. We can argue now that the feedback, or absolute, instability can emerge in a slowly varying incompressible boundary-layer flow as a result of coupling between quasi-parallel modes from discrete and continuous spectra.

Qualitative features such as a discrete spectrum of feedback modes and the growing number of modes under increase of the total length parameter (in this paper - the length $L$ ) are in agreement with the results for other systems; see, e.g., [9] for a flow governed by a mixed hyperbolic/parabolic system of equations and with exponential decay in upstream influence. However, the conditions for the origin of feedback under elliptic mechanisms of upstream influence seem less restrictive as they only require a sufficiently large value of $L$.

Acknowledgements. Fruitful discussions with Professors S.N. Brown and F.T. Smith and the suggestions made by the referees are gratefully acknowledged.

\section{References}

1. J.-M. Chomaz, P. Huerre and L. G. Redekopp. A frequency selection criterion in spatially developing flows. Stud. Appl. Math. 84 (1991), 119-144.

2. P. Huerre and P. A. Monkewitz. Local and global instabilities in spatially developing flows. Ann. Rev. Fluid Mech. 22 (1990), 473-537.

3. A. P. Rothmayer and F. T. Smith. Incompressible triple-deck theory. In The Handbook of Fluid Dynamics, ed. R. Johnson, Springer (1998).

4. O. S. Ryzhov and E. D. Terent'ev. On the transition regime characterising the start of a vibrator in a subsonic boundary layer on a plate. Prikl. Matem. Mekh. 50(6) (1986), 974-986.

5. F. T. Smith. On the non-parallel flow stability of the Blasius boundary layer. Proc. Roy. Soc. Lond. 366 (1979), 91-109.

6. F. T. Smith and R. J. Bodonyi. On short-scale inviscid instabilities in flow past surfacemounted obstacles and other non-parallel motions. Aero. J. June/July (1985), 205-212.

7. K. Stewartson. Multi-structured boundary layers on flat plates and related bodies. Adv. Appl. Mech. 14 (1974), 145-239.

8. V. V. Sychev, A. I. Ruban, ic. V. Sychev and G. L. Korolev. Asymptotic Theory of Separated Flows. Cambridge University Press (1998).

9. S. N. Timoshin and F. T. Smith. Non-local interactions and feedback instability in a high Reynolds number flow. Theoret. Comp. Fluid Dyn., submitted (2000).

10. O. R. Tutty and S. J. Cowley. On the stability and the numerical solution of the unsteady interactive boundary-layer equation. J. Fluid Mech. 168 (1986), 431-456.

11. V. I. Zhuk and O. S. Ryzhov. Free interaction and stability of an incompressible boundary layer. Dokl. Akad. Nauk USSR 263 (1980), 56-59 (in Russian).

Dr. S. N. Timoshin,

Department of Mathematics,

University College London,

Gower Street, London WC1E 6BT
MSC (2000): Primary, 76E09

Received on the 21st of November, 2002. 